EPJ manuscript No.

(will be inserted by the editor)

\title{
High $\mathrm{p}_{\mathrm{T}}$ Jets and Photons at the Tevatron
}

\author{
Cecilia E. Gerber ${ }^{\mathrm{a}}$ \\ University of Illinois at Chicago, e-mail: gerber@uic.edu \\ Received: date / Revised version: date
}

\begin{abstract}
I present recent results on high $p_{\mathrm{T}}$ jets and photon production in $\bar{p} p$ collisions at a center of mass energy of $1.96 \mathrm{TeV}$. The measurements were performed by the CDF and D $\varnothing$ collaborations using between 150 and $300 \mathrm{pb}^{-1}$ of data taken during Run II at the Tevatron.
\end{abstract}

PACS. 13.87.Ce 12.38.Qk 13.85.Qk

\section{Introduction}

Quantum Chromodynamics (QCD) emerged as a mathematically consistent theory in the 1970 s, and nowadays is regarded as one of the cornerstones of the Standard Model. One of the triumphs of modern particle physics has been the extent to which QCD has successfully accounted for the strong interaction processes observed experimentally at hadron colliders. Recent measurements of multi-parton processes, heavy flavor production, and direct photon production are pushing the comparisons with available theoretical models to a new era of precision QCD studies. This confrontation between measurements and predictions is important in itself as a test of QCD, but is also crucial in the identification of beyond the standard model phenomena that might appear at the Tevatron or the LHC. In the following sections I will present recent results from the Tevatron collider experiments on high $p_{\mathrm{T}}$ jets and photon production.

\section{Dijet Azimuthal Decorrelations}

The D $\varnothing$ collaboration has recently published [1] a study of the correlations in the azimuthal angle between the two largest transverse momentum jets $\left(\Delta \Phi_{\text {dijet }}\right)$ using 150 $\mathrm{pb}^{-1}$ of data collected during Run II. Dijet production in hadron-hadron collisions in the absence of radiative effects results in two jets with equal transverse momenta and correlated azimuthal angles. Additional soft radiation causes small azimuthal decorrelations, but $\Delta \Phi_{\text {dijet }}$ values significantly lower than $\pi$ are evidence of additional hard radiation with high $p_{\mathrm{T}}$. Exclusive 3 -jet production populates the region of $\Delta \Phi_{\mathrm{dijet}}$ between $2 \pi / 3$ and $\pi$, while smaller values of $\Delta \Phi_{\text {dijet }}$ require additional radiation such as a fourth jet in the event. Distributions of $\Delta \Phi_{\text {dijet }}$ consequently provide an ideal testing ground for higher-order

\footnotetext{
a for the CDF and DØ Collaborations
}

pQCD predictions without requiring the reconstruction of additional jets.

The analysis is based on an inclusive dijet sample in the central rapidity region $(|y|<0.5)$, and was performed in four analysis regions, defined by the requirement that the $p_{\mathrm{T}}$ of the leading jet in the event $p_{\mathrm{T}}^{\max }$ be greater than $75,100,130$, and $180 \mathrm{GeV}$, respectively. The second leading jet in the events was required to have $p_{\mathrm{T}}>$ $40 \mathrm{GeV}$. Jets were defined using an iterative seed-based cone algorithm (including mid-points) with radius $R_{\text {cone }}=$ 0.7 [2]. The observable was defined as the differential dijet cross section in $\Delta \Phi_{\text {dijet }}$, normalized by the dijet cross section integrated over $\Delta \Phi_{\text {dijet }}$ in the same phase space $\left(1 / \sigma_{\text {dijet }}\right)\left(d \sigma_{\text {dijet }} / d \Delta \Phi_{\text {dijet }}\right)$. The data was compared to pQCD calculations obtained using the parton-level event generator NLOJET++ [3] and CTEQ6.1M [4] PDF's. The renormalization and factorization scales were chosen to be $\mu_{r}=\mu_{f}=0.5 p_{\mathrm{T}}^{\max }$. It is observed that NLO pQCD provides a good description of the data. In addition, the data was compared to Monte Carlo event generators (HERWIG [5] and PYTHIA [6]), that use $2 \rightarrow 2$ LO pQCD matrix elements with phenomenological parton-shower models to simulate higher order QCD effects. HERWIG version 6.505 describes the data well over the entire range, whereas PYTHIA version 6.225 with default parameters describes the data poorly. A good agreement can be achieved between data and PYTHIA by increasing the maximum allowed virtuality by a factor of four [7]. The data can therefore benefit global efforts to tune Monte Carlo event generators.

\section{3 b Jet Cross Sections}

Measurements of the $b$ jet cross section provide an important quantitative test of $\mathrm{pQCD}$, as the mass of the $b$ quark is considered to be large enough to justify the perturbative expansion in the strong coupling constant. Data on $b$ quark production is therefore expected to be adequately 
described by NLO pQCD calculations. Both the CDF and the $\mathrm{D} \emptyset$ collaborations have recently made public preliminary results on inclusive $b$ jet cross section measurements. These results extend the upper reach of exclusive measurements using $B$ mesons, and provide a simple observable with high sensitivity to heavy flavor production up to the highest $p_{\mathrm{T}}$. In addition, theoretical uncertainties on fragmentation and decay are smaller in the inclusive $b$ jet analysis than in exclusive decay studies. In the following sub-sections, I will briefly describe the methods used by CDF and D $\varnothing$ to identify $b$ jets, as well as present preliminary results on inclusive $b$ jet production.

\section{1 b Jet Identification}

Two strategies have been developed to identify jets that originate from a $b$ quark, refered to as $b$ tagging: softlepton tagging (SLT) and secondary vertex tagging ( $\mathrm{SecVtx}$ )

Approximately $20 \%$ of the time, a decaying $b$ quark will yield a muon, either directly or through a sequential decay via a $c$ quark. These muons are much softer and less isolated than the ones originating from a $W$ boson, and when identified within the jet cone, can be used to tag that jet as originating from a $b$ quark. Although the fraction of $b$ quarks decaying into electrons is the same as for muons, the identification of soft electrons inside jets is quite challenging and is not being considered further in this paper.

Another method of tagging $b$ jets profits from the relatively long lifetime of $B$ hadrons, which allows them to travel up to several $m m$ before decaying. The SecVtx algorithm [8] relies on the displacement of secondary vertices relative to the primary event vertex to identify $B$ hadrons. It uses displaced tracks associated with a jet that are within a sub-cone of 0.4 in the $\eta-\phi$ space with respect to the jet axis. The algorithm looks for combinations of at least 2 tracks consistent with originating from a secondary vertex. For each secondary vertex, the distance in the transverse plane between the vertex and the primary one ( $L_{x y}$ or decay lenght) is calculated, and tags with positive $L_{x y}$ are accepted as $b$ tags.

\subsection{High $\mathrm{p}_{\mathrm{T}}$ Cross Section for $\mu$ tagged Jets}

The $\mathrm{D} \emptyset$ collaboration has measured the inclusive jet cross section for $\mu$ tagged jets using $294 \mathrm{pb}^{-1}$ of data collected during Run II. Events were recorded using single jet triggers at four different threholds; the highest $p_{\mathrm{T}}$ threshold trigger being unprescaled. Jets were defined using an iterative seed-based cone algorithm (including mid-points) with radius $R_{\text {cone }}=0.5$ [2], and were restricted to central rapidities of $|y|<0.5$. Muons were detected as tracks reconstructed from hits recorded in three layers of tracking detectors and two layers of scintillators [9], both located outside the calorimeter. A 1.8 Tesla iron toroidal magnet is located outside the innermost layer of the muon detector. The $p_{\mathrm{T}}$ of the muon was required to be greater than $5 \mathrm{GeV}$, and the distance in $\eta-\phi$ space between the muon and the jet was required to be less than 0.5 , which selects a sample enriched in heavy flavor jets. The data was then fully corrected for efficiencies and unsmeared to particle level. The selected sample contains contributions from $b$ and $c$ quark semileptonic decays, but also from in flight decays of $\pi$ and $K$ mesons. To extract the heavy flavor component of the $\mu$ tagged jets cross section $\mathrm{D} \emptyset$ used a sample of QCD Monte Carlo events generated with PYTHIA and processed with a GEANT-based [10] full detector simulation. The fraction of $\mu$ tagged jets which contain at least one $b$ or $c$ quark as predited by the Monte Carlo is shown in Fig. 1 (left). The overlaid fit represents the fraction used as a correction factor to the measured cross section. It ranges from about $70 \%$ at $p_{\mathrm{T}}=50 \mathrm{GeV}$ to about $45 \%$ at $p_{\mathrm{T}}=400 \mathrm{GeV}$. The dashed lines represent the assigned systematic uncertainty on the fraction, which reflects the limited Monte Carlo statistics, will be reduced for a future update of the analysis. Fig. 1 (right) . shows the comparison of the data with theoretical predictions. The result is presented as a ratio with the prediction from PYTHIA for the $\mu$ tagged jets cross section originating from $b$ or $c$ quarks. PYTHIA's prediction therefore is shown as a line at 1 . The data is shown as dots with statistical error. The systematic error is shown as a band: the outer band corresponds to the total error, the middle band corresponds to setting the heavy-flavor fraction error to zero, and the inner band shows the exclusive contribution to the error from the jet energy scale correction. The NLO prediction was obtained from the product of the predicted inclusive jet cross section from NLOJET ++ with CTEQ6M and $\mu=p_{\mathrm{T}} / 2$, multiplied by the fraction of jets from $b$ or $c$ quarks that are $\mu$ tagged, as predicted by PYTHIA. The data lays approximately between the two calculations, with errors spanning the difference. Further reduction of the experimental uncertainties is needed in order to constrain the predictions.

\subsection{Inclusive b Jet Cross Section}

The CDF Collaboration has measured the inclusive $b$ jet cross section using about $300 \mathrm{pb}^{-1}$ of data collected during Run II. Events were recorded using single jet triggers at five different threholds, optimized to provide unbiased, fully efficient data selection. Jets were defined using an iterative seed-based cone algorithm (including mid-points) with radius $R_{\text {cone }}=0.7$, and were restricted to central rapidities of $|y|<0.7$. The SecVtx algorithm was used to tag $b$ jets. Monte Carlo was used to derive the average correction for detector effects and $p_{\mathrm{T}}$ scale. The $b$ tagging efficiency was measured in a Monte Carlo dijet sample, and scaled by a Data-to-Monte Carlo correction factor derived from comparing the $b$ tagging efficiency obtained from an inclusive electron data sample and the corresponding Monte Carlo sample. To extract the heavy flavor component of the $b$ tagged jets cross section, CDF used the shape of the SecVtx vertex mass distribution as the discriminating quantity. Templates for $b, c$, or light-quark jets were obtained from PYTHIA Monte Carlo in bins of jet $p_{\mathrm{T}}$; the templates for $c$ and light jets were subse- 

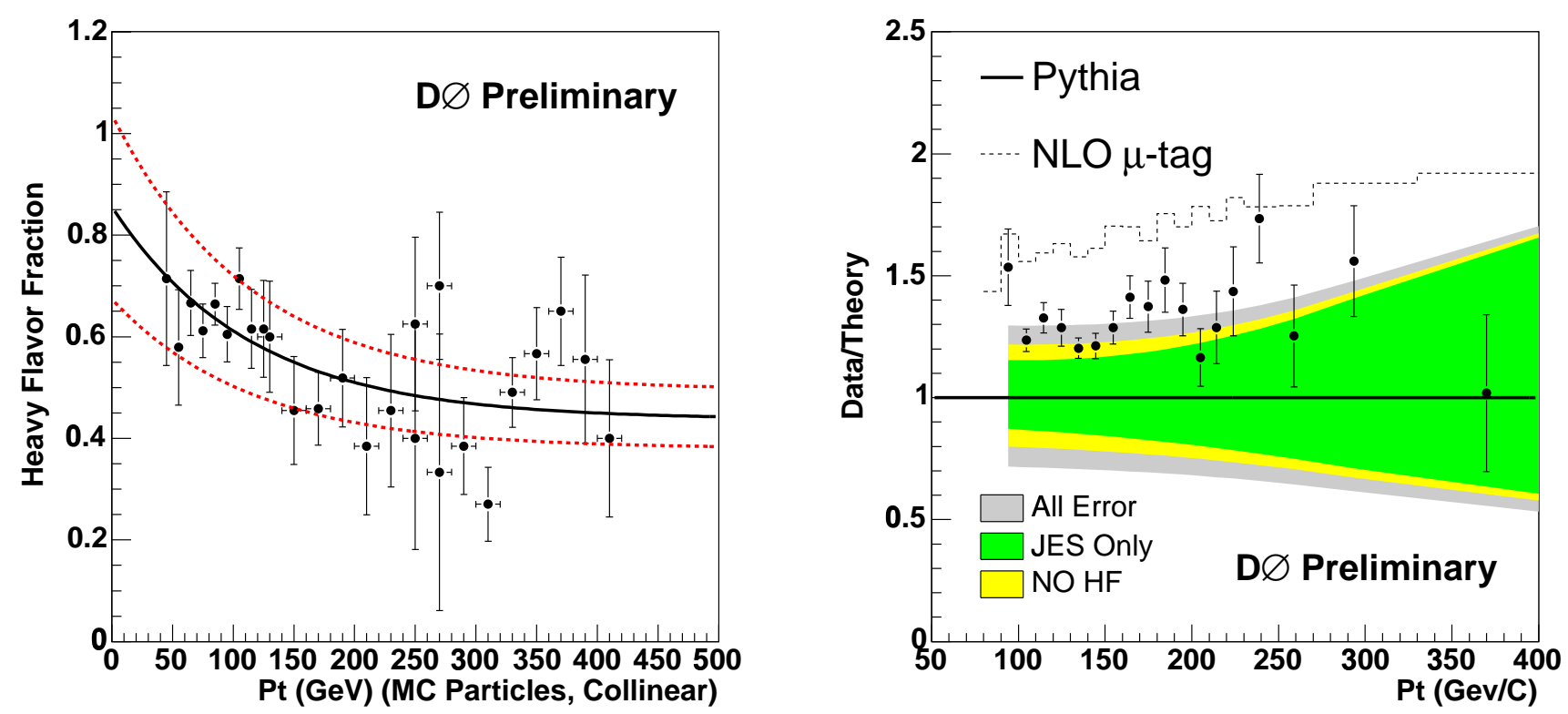

Fig. 1. Fraction of $\mu$ tagged jets coming from $b$ or $c$ quark semileptonic decays as predicted by PYTHIA (left). D $\varnothing$ measurement of the inclusive cross section for $\mu$ tagged $b$ or $c$ quark jets presented as a ratio with the prediction by PYTHIA, compared to the same PYTHIA prediction, and to a NLO estimate obtained as described in the text (right).

quently merged. The fraction of tagged jets that originate from a $b$ quark in each jet $p_{\mathrm{T}}$ bin was obtained by fitting the data to a linear combination of the templates for $b$ and $c$ or light-quark jets. The obtained fraction can be seen in Fig. 2 (left). The measured inclusive $b$ jet cross section is shown in Fig. 2 (right), together with the prediction from PYTHIA Tune A, with CTEQ5L PDF. The error in the last six bins is dominated by the error on the fraction of $b$ jets and the absolute jet energy scale. PYTHIA underestimates the data by a factor of 1.4 , as expected for a $\mathrm{LO}$ Monte Carlo prediction. A comparison of the data with NLO QCD is in preparation.

\section{Photon Studies}

At the Tevatron, the dominant source for production of photons with $p_{\mathrm{T}} \leq 150 \mathrm{GeV}$ is through the process $q+q \rightarrow$ $q+\gamma$ (Compton Scattering). The production cross section is therefore sensitive to the gluon density inside the colliding hadrons. The measurement of the isolated photon cross section allows testing of NLO and resummed QCD calculations, as well as phenomenological models of gluon radiation, photon isolation, and the fragmentation process. In addition, diphoton final states serve as signature for many interesting physics processes [11], including one of the main discovery channels for the Higgs boson at the LHC [12]. Nevertheless, the QCD production rate dominates, and thus an understanding and modelling of the QCD production mechanism is needed pior to any possible discoveries.

\subsection{Isolated Photon Cross Section}

The $\mathrm{D} \emptyset$ collaboration has measured the inclusive cross section for the production of isolated photons using 326 $\mathrm{pb}^{-1}$ of data collected during Run II. The preliminary result has been shown for the first time at the Hadron Collider Physics Symposium 2005 (this conference), and is presented below. Events were required to pass a combination of unprescaled EM triggers. Photons were reconstructed with a simple cone algorithm [13] with cone size $R_{\text {cone }}=0.2$, had a minimum $p_{\mathrm{T}}^{\gamma}$ of $15 \mathrm{GeV}$, and were restricted to central rapidities of $|y|<0.9$. Each photon candidate was required to deposit more than $95 \%$ of the detected energy in the EM section of the calorimeter, and to be isolated in an annular region between $R_{\text {cone }}=0.2$ and 0.4 around the photon. In addition, the probability to have a track spatially matched to the EM cluster was required to be less than 0.001 , and the missing transverse energy $\left(\mathbb{E}_{T}\right)$ had to satisfy the cut $\mathbb{E}_{T} / p_{\mathrm{T}}^{\gamma}<0.7$. A set of four additional variables that are well modelled in Monte Carlo were used to build an artificial neural network (ANN) [14] optimized for pattern recognition. The network is trained in bins of $p_{\mathrm{T}}^{\gamma}$ to produce an output of unity for signal and zero for background. The normalized distribution of ANN output for data, direct photon signal Monte Carlo and a background sample of electromagnetic jets obtained directly from collider data, is shown in Fig. 3 (left) for the bin $44<p_{\mathrm{T}}^{\gamma}<50 \mathrm{GeV}$. Events that satisfy a cut in the $\mathrm{NN}$ output of greater than 0.5 remain in the final data sample. The purity of this sample is determined on a statistical basis by fitting the data to a linear combination of the ANN distributions for direct photon Monte Carlo (signal), and electromagnetic jets from data (background). 

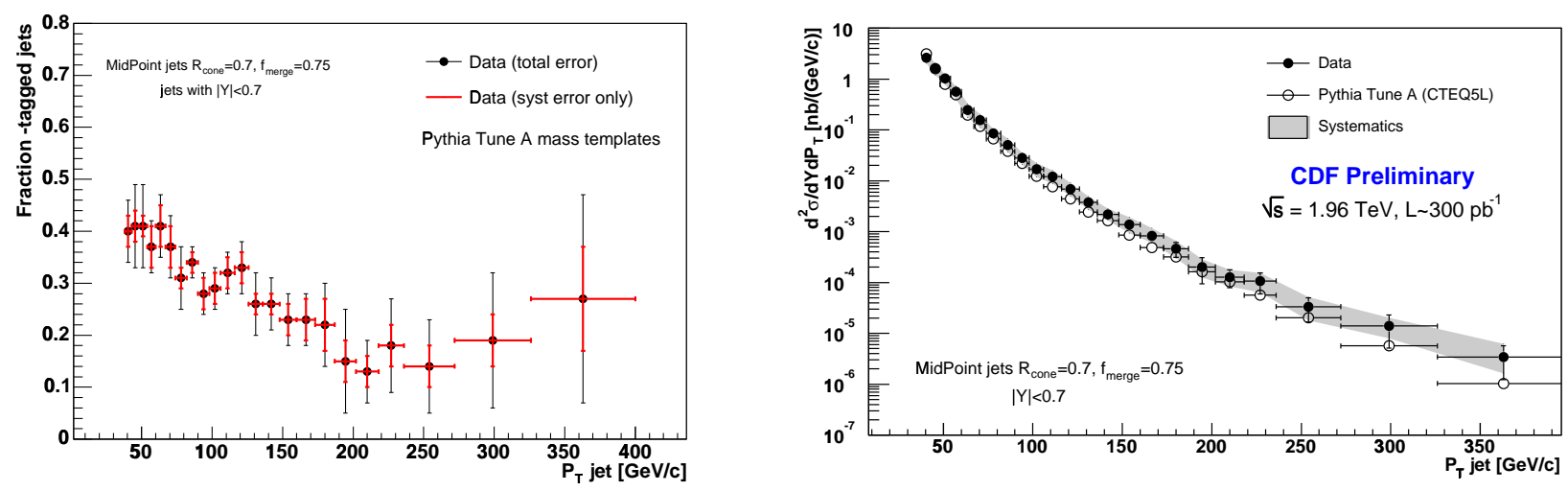

Fig. 2. Fraction of SecVtx tagged jets coming from a $b$ quark as obtained from fits to the vertex mass distribution predicted by PYTHIA (left). CDF measurement of the inclusive $b$ jet cross section compared with a prediction from PYTHIA TUNE A, with CTEQ5L PDF (right).

The result is shown in Fig. 3 (right). The inclusive photon cross section was obtained by the relation

$$
\frac{d^{2} \sigma}{d p_{\mathrm{T}}^{\gamma} d \eta_{\mathrm{T}}^{\gamma}}=\frac{N P f}{L \Delta p_{\mathrm{T}}^{\gamma} \Delta \eta_{\mathrm{T}}^{\gamma} A \varepsilon}
$$

where $N$ is the number of selected photon candidates, $P$ is the photon purity, $f$ is the unsmearing correction, $L$ is the integrated luminosity, $\Delta p_{\mathrm{T}}^{\gamma}$ and $\Delta \eta_{\mathrm{T}}^{\gamma}$ are the bin sizes in transverse momentum and pseudorapidity of the photon respectively, $A$ is the acceptance, and $\varepsilon$ is the efficiency of the selection. The ratio of the measured cross section to the NLO QCD prediction [15] is shown in Fig. 4 . The prediction agrees with the data within experimental uncertainties in the whole considered range $23<p_{\mathrm{T}}^{\gamma}<300 \mathrm{GeV}$. The prediction by Gordon and Vogelsang [16], that uses a different set of fragmentation functions, is in agreement within $7 \%$.

\subsection{Diphoton Production}

The CDF collaboration has recently published [17] a measurement of the production cross section for isolated prompt diphotons using $207 \mathrm{pb}^{-1}$ of data collected during Run II. The experimental result was compared to three prediction: DIPHOX [18], ResBos [19], and PYTHIA [6]. DIPHOX is a fixed-order QCD calculation that includes all subprocesses at NLO. ResBos includes subprocesses where the two photons are produced at the hard-scattering at NLO, and fragmentation contributions at LO, but resummation is used to include the effects of initial state gluon radiation. PYTHIA is a parton shower Monte Carlo that contains the processes at LO. Several distributions were examined: the diphoton mass $\left(m_{\gamma \gamma}\right)$, photon transverse momentum $\left(p_{\mathrm{T}}^{\gamma}\right)$, and the azimuthal angle between the 2 photons $(\Delta \Phi)$. The predictions for the $m_{\gamma \gamma}$ distribution agree fairly well with data, except for the very low mass region, where DIPHOX predicts a higher rate. Because of high $g g$ collision luminosity at low $m_{\gamma \gamma}$, the $g g$ subprocess provides the greatest contribution to the cross section

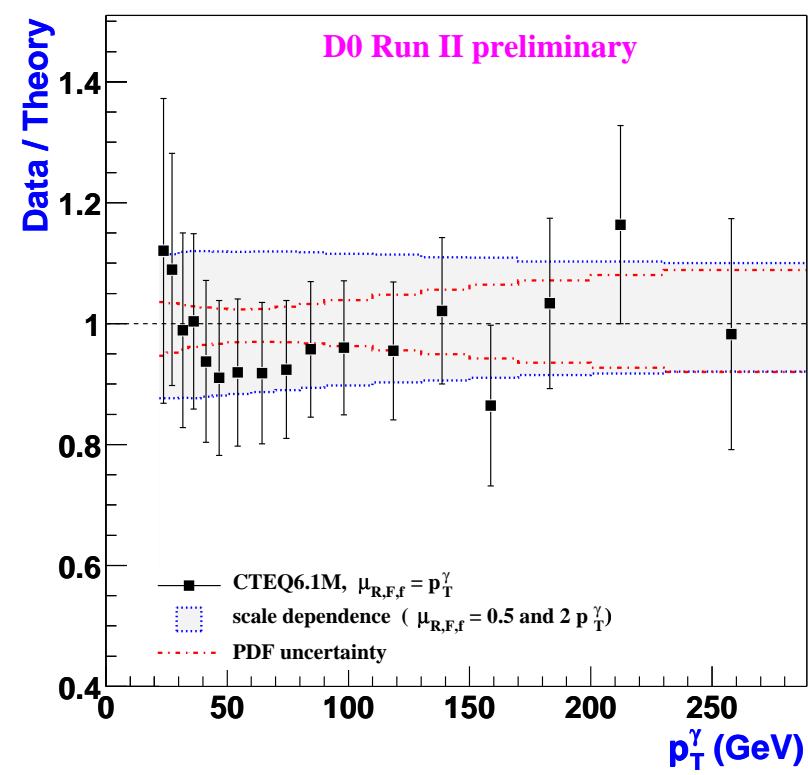

Fig. 4. Ratio of the measured isolated photon cross section to the NLO QCD [15]. Variations due to the choice of scales and PDF uncertainties are shown as blue and red lines, respectively.

in that region. Low mass $\gamma \gamma$ production therefore serves as an interesting arena for examining production from a $q q$ initial state in preparation for Higgs searches at the LHC. The ResBos prediction for the $p_{\mathrm{T}}^{\gamma}$ distribution provides a smooth description over the entire range, while the DIPHOX curve is unstable at low $p_{\mathrm{T}}^{\gamma}$ due to the divergence of the fixed-order QCD calculations when $p_{\mathrm{T}}^{\gamma} \rightarrow 0$. For the $\Delta \Phi$ distribution, the ResBos curve lies above the DIPHOX prediction at values close to $\pi / 2$, but lies significantly below the DIPHOX curve at small $\Delta \Phi$. Overall, the differences observed between the predictions are as expected. 

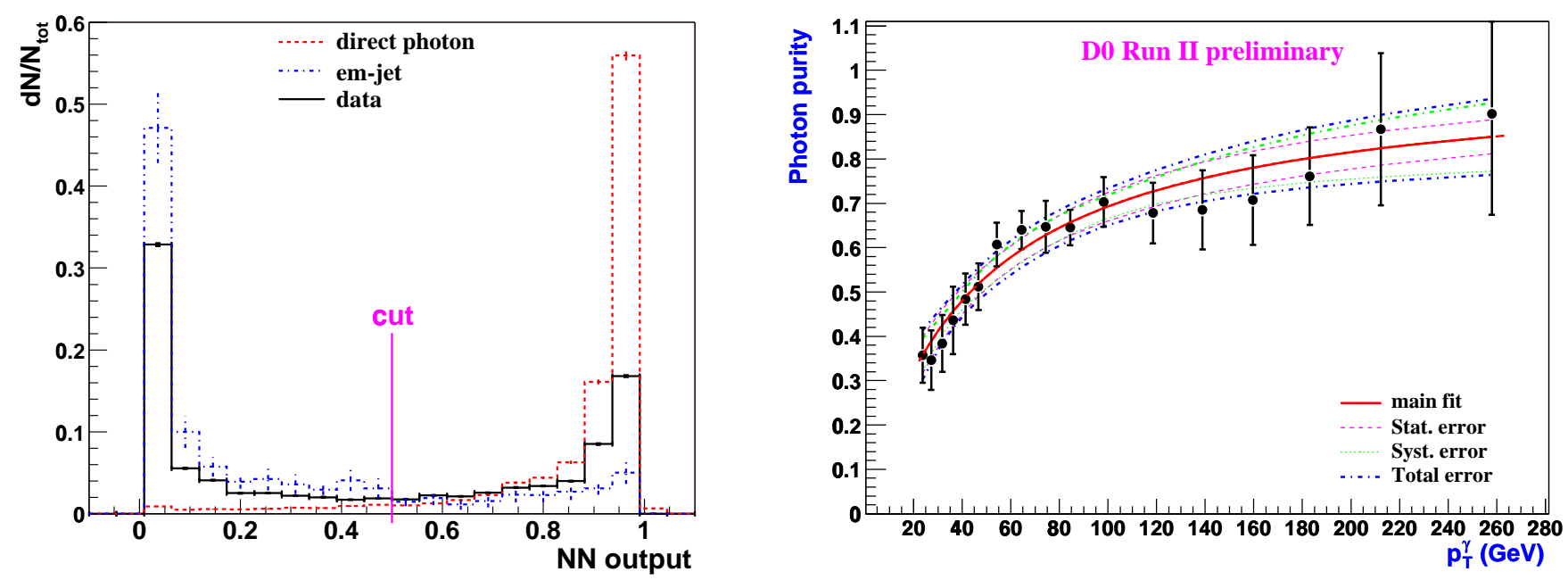

Fig. 3. Normalized distributions of ANN output for data, signal, and background events for the bin of $44<p_{\mathrm{T}}^{\gamma}<50 \mathrm{GeV}$ (left). Photon purity as determined from a fit to the ANN output shown together with the chosed purity parametrization and the assigned uncertainty (right).

\section{Conclusions}

I presented recent measurements on dijet azimuthal decorrelations, inclusive heavy-flavor cross sections and photon studies using between 150 and $300 \mathrm{pb}^{-1}$ of recent data recorded by the Tevatron hadron collider experiments. General agreement is observed between data and NLO QCD. The experimental results are approaching a precision that allows detail comparisons with different theoretical prediction and tuning of Monte Carlo event generators. Understanding QCD to the fullest extend is not only important in itself, but also crucial for many precision Standard Model measurements and searches for new physics.

\section{Acknowledgments}

I would like to thank my collaborators from the CDF and $\mathrm{D} \varnothing$ collaborations for their help in preparing this document. I also thank the staffs at Fermilab and collaborating institutions, and acknowledge support from the National Science Foundation (USA).

\section{References}

1. V. M. Abazov et al., The D $\varnothing$ Collaboration, Phys. Rev. Lett.94, (2005) 221801.

2. G. C. Blazey et al., in Proceedings of the Workshop: QCD and Weak Boson Physics in Run II, edited by U. Baur, R. K. Ellis, and D. Zeppenfield, Batavia, Illinois (2000) p. 47. See Section 3.5 for details.

3. Z. Nagy, Phys. Rev. Lett.88, (2002) 122003.

4. J. Pumplin et al., JHEP 0207, (2002) 12; D. Stump et al., JHEP 0310, (2003) 046.

5. G. Marchesini et al., Comput. Phys. Commun. 67, (1992) 465; G. Corcella et al., JHEP 01, (2001) 010.
6. T. Sjöstrand et al., Comput. Phys. Commun. 135, (2001) 238.

7. The PYTHIA parameter $\operatorname{PARP}(67)$ was increased from the default of 1.0 to 4.0 .

8. T. Affolder et al., Phys. Rev. D64, (2001) 032002.

9. V. Abazov et al., Fermilab-PUB-05-034-E.

10. R. Brun and F. Carminati, CERN program library long writeup W5013 (1993).

11. B. Abbott et al.(The D $\varnothing$ Collaboration), Phys. Rev. Lett.86, (2001) 1156. G. F. Giudice and R. Rattazzi, Phys. Rep. 322, (1999) 41.

12. ATLAS Collaboration, LHCC/P2 (1994), and LHCC/9915 (1999). CMS Collaboration, LHCC/P1 (1994).

13. V. Abazov et al.(The D $\varnothing$ Collaboration), Phys. Rev. Lett.textbf87, (2001) 251805.

14. C. Peterson et al., "JETNET 3.0. A versatile Artificial Neural Network Package", Lund University Preprint LU-TP 93-29.

15. P. Aurenche et al., JETPHOX package; S. Catani et al., JHEP textbf0205,(2002) 028.

16. L. E. Gordon and W. Vogelsang, Phys. Rev. Dtextbf 48 , (1993) 3136 and Phys. Rev. Dtextbf50, (1994) 1901.

17. D. Acosta et al., The CDF Collaboration, hep-ex/0412050, accepted by Phys. Rev. Lett..

18. T. Bonoth et al., Eur. Phys. J. C textbf16, (2000) 311.

19. C. Balasz et al., Phys. Rev. Dtextbf57, (1998) 6934. 